\title{
An energy-efficient clustering protocol using fuzzy logic and network segmentation for heterogeneous WSN
}

\author{
Aziz Mahboub ${ }^{1}$, El Mokhtar En-Naimi ${ }^{2}$, Mounir Arioua ${ }^{3}$, Hamid Barkouk $^{4}$, Younes El Assari ${ }^{5}$, Ahmed \\ El Oualkadi ${ }^{6}$ \\ ${ }^{1,2,4}$ LIST laboratory Department of Computer Sciences, Faculty of Sciences and Technologies, Abdelmalek Essaadi \\ University, Morocco \\ ${ }^{3}$ National School of Applied Sciences, Abdelmalek Essaadi University, Morocco \\ ${ }^{5,6}$ LabTIC Laboratory, National School of Applied Sciences, Abdelmalek Essaadi University, Tangier, Morocco
}

\begin{tabular}{l}
\hline \hline Article Info \\
\hline Article history: \\
Received Jan 19, 2019 \\
Revised Apr 1, 2019 \\
Accepted Apr 19, 2019 \\
\hline Keywords: \\
Energy efficiency \\
WSN segmentation \\
Network lifetime \\
Energy consumption; Routing \\
protocols \\
Clustering \\
Fuzzy means \\
Subtractive clustering method
\end{tabular}

\begin{abstract}
Wireless sensor networks have become an emerging research area due to their importance in the present industrial application. The enlargement of network lifetime is the major limitation in WSN. Several routing protocols study the extension of lifespan in WSN. Routing protocols significantly influence on the global of energy consumption for sensors in WSN. It is essential to correct the energy efficiency performance of routing protocol in order to improve the lifetime. The protocols based on clustering are the most routing protocols in WSN to reduce energy consumption. The protocols dedicate to WSN have demonstrated their limitation in expanding the lifetime of the network. In this paper, we present Hybrid SEP protocol : Multi-zonal Fuzzy logic heterogeneous Clustering based on Stable Election Protocol (FMZ-SEP). The FMZSEP characterizes by four parameters: WSN segmentation (splitting the WSN into the triangle zones ), the Subtractive Clustering Method to determine a correct number of clusters, the FCM and the SEP protocol. The FMZ-SEP prolong the stability period and extend the lifetime. The simulation results point out that the stability period of FMZ-SEP. FMZ-SEP protocol outperforms of MZ-SEP, FSEP and SEP protocol by improving the network lifetime and the stability period.
\end{abstract}

Copyright (C) 2019 Insitute of Advanced Engineeering and Science. All rights reserved.

\section{Corresponding Author:}

Aziz Mahboub,

LIST laboratory Department of Computer Sciences,

Faculty of Sciences and Technologies, Abdelmalek Essaadi University,

B.P 410, Route de Charf, Tangier, Morocco.

Phone : +212666145279

Email : amahboub@uae.ac.ma

\section{INTRODUCTION}

In the last ten years, researchers have shown interest in WSN. Divers domains use WSN to improve their production or quality of service, like smart city, smart roads, smart lighting,etc. The main functions in WSN are collected data, processing and broadcasting for different environments and applications [1, 2, 3].

WSN constitutes by a large numbers of small devices that communicate with each other via radio links for information sharing and cooperative treatment. These devices can be randomly deployed in an area of interest to supervise or monitor various phenomena $[4,5]$. The sensor node works separately without any central control; a malfunctions of some sensor nodes does not interfere with the operation of WSN.

The sensors nodes send collected data to the base station (BS) in multi-hop mode by means of $\mathrm{CH}$ or mono-hop mode [6]. Typically, the sensor node is a tiny device that is equipped with a transducer for data acquisition, a microcomputer for local data processing and storage, a transceiver for data transmission 
and reception and power source; It is powered up by a limited battery which is mostly impossible to change $[7,8,9]$. The WSN manage by one BS or more; which collects data for processing and sending the data to the datacenter [10].

The WSN have high density of nodes , therefore a large quantity of energy is consumed in the routing. This requires an optimization of energy consumption in the routing [8]. Various works studied routing protocols to improve the network's lifetime. Routing protocols in WSN grouped into flat routing, clustering routing and location routing $[10,11]$.

In the clustering protocols, the nodes are required to classify in non-overlapping clusters with each set possessing a Cluster Head $(\mathrm{CH})$. The main function of $\mathrm{CH}$ is aggregating and transmitting data to the BS; which can be connected to a data-center by the internet or a satellite [7,8]. The protocol based on clustering optimizes consumption of energy compared to other protocols . It is practical for the large WSN. Because the clustering protocol manage only the $\mathrm{CH}$ not the entire WSN [11, 12].

In this work, we focus on the clustering protocols. we propose the hybrid of MZ-SEP protocol called MZF-SEP. The proposed protocol remarkably improve the performance parameters of WSN like lifetime and stability period. The MZF-SEP protocol segment the WSN on the multiple triangle zone to allow provides appropriately a correct $\mathrm{CH}$ repartition in the network. The MZF-SEP protocol uses FCM with SEP protocol in the zone that are very far from the BS. The zone near of the BS work only the SEP protocol. The MZF-SEP protocol improve energy-effecient of member's cluster. In addition the MZF-SEP protocol works in random distribution of nodes or in uniform distribution of sensor nodes. The MZF-SEP has proved considerable minimization of sensor nodes energy consumption and significant extension of the network lifetime.

This paper is organized as follows: The related work is presented in section 2. In section 3 we present MZF-SEP protocol. The performance parameters and simulation results are presented in section 4. Finally, conclusions are drawn in section 5 .

\section{RELATED WORK}

Several clustering algorithms solutions are proposed for WSN, these are proposed deal two key point: To manage the routing and the data processing appropriately in order to achieve energy savings in the WSN. In this part, we describe some of the better performers routing algorithms specifically designed for WSN.

The authors in [13] proposed the Stable Election Protocol (SEP). The SEP protocol implemented two level heterogeneous, the nodes are classified into two types according to the initial energy quantity $[14,15]$. The first group is comprised by the nodes contain more energy compared to other nodes. these nodes which call advanced nodes. The percentage of supplementary energy of the advance nodes in relation to the normal node is denoted by $\beta$ therefore The advanced nodes have $(1+\beta)$ more energy compared to the normal nodes.

Advanced nodes can have more probability to emerge as CH than normal nodes [16]. The SEP protocol applies the principle of LEACH protocol for the selection of $\mathrm{CH}$, the election operation of $\mathrm{CH}$ is based on weighted election probabilities of each node to be converted to a $\mathrm{CH}$ [10]. The SEP protocol adopts two weighted election probability $P_{n o r m a l}$ and $P_{a d v} . P_{a d v}$ is intended for the advanced nodes. Pnormal is destine for the normal nodes [13].

In [17], the authors proposed new approach named Threshold Sensitive Stable Election Protocol (TSEP) based on SEP protocol. The TSEP protocol classified the nodes in three levels of heterogeneity according to the energy levels: normal nodes, intermediate nodes and advanced nodes.

The intermediate nodes have energy levels more than the initial energy of normal node and less than the initial energy of advanced nodes. Each type of node has an optimal probability and its threshold value. The TSEP improve stability period and network life than SEP and TEEN [18].

The MZ-SEP protocol is improved version of the SEP protocol. The MZ-SEP is properly partitioned into triangle zones and provides appropriately a well cluster repartition in the network in order to improve the stable period of the networks, and the lifetime of network [11]. Figure 1 present the network architecture of MZ-SEP protocol.Zones creation is based on the following parameters:

(a) The position of the base station;

(b) The value of $d_{0}$;

(c) The deployment area dimensions. 


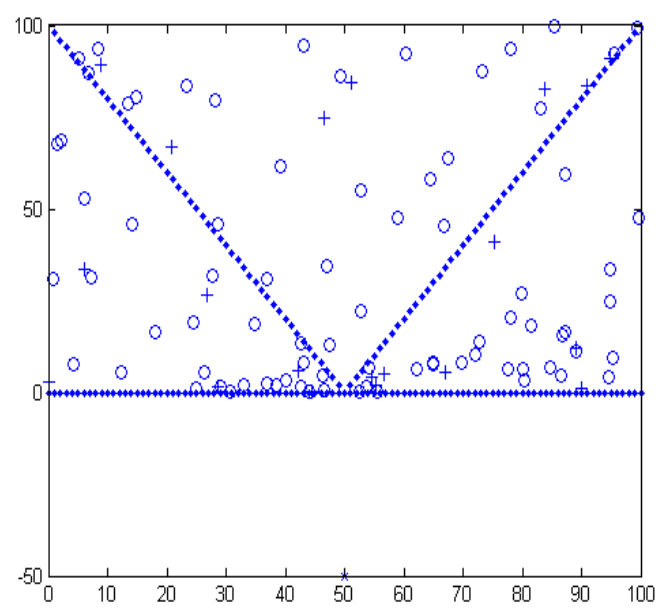

Figure 1. Network architecture in MZ-SEP [11]

MZ-SEP operate by making the position more than $50 \%$ of $C H$ as close as possible to the $B S$. The member nodes must be attached to the closest $\mathrm{CH}$ in the network. knowing that the distance between the numerous $C H$ generated by MZ-SEP and the base station is less than or equal to $d_{0}$. The MZ-SEP protocol only attempts to find the global minimum between cluster head and its members.

\section{CLASSIFICATION METHODS}

This section briefly describes the various classification methods used in order to create clusters. The methods used are Fuzzy C-mean (FCM) algorithm and Subtractive Clustering Algorithm.

\subsection{Subtractive clustering method}

The algorithms based on clustering need the user to prespecify the number of cluster centers and their initial locations. The quality of the solution relating to strongly on the choice of initial values as the number of cluster centres and their initial locations.

The identification of the optimal number of clusters isdifficult to do. The results depending on the way of distribution the sensor nodes in the field and the desired clustering resolution of the user. Cluster analysis can help to identifying the number optimal of clusters $[19,20,21]$.

In 1995, the author proposed an upgraded version of the mountain method [19], titled the subtractive method, in which each element is considered as a potential cluster centroid. Consider the following a collection of $N$ nodes $\{x 1, x 2 \ldots x n\}$ in sensor area. All sensor node has a possibility to become a cluster centre, which can be denoted as $C H$ the potentiality of sensor node $x_{i}$ [22].

$$
M(x)=\sum_{j=1}^{n} e^{-\beta\left\|x_{i}-x_{j}\right\|}
$$

Where, $\beta$ is a positive constant and $\left\|x_{i}-x_{j}\right\|^{2}$ is the square of the distance between the node $x_{i}$ and the node $x_{j}$. Using this mountain function, the upgraded version of the mountain method adopte same mécanisme used in the original mountain method to selected the cluster centroids[23]. $M_{1}^{*}$ be the maximum value of the mountain function.

$$
M_{1}^{*}=\max _{i}\left\|x_{i}\right\|
$$

Where, $x_{i}$ is the node in WSN whose mountain value is $M_{1}^{*}$; this node is selected as the first cluster centroid. 


\subsection{Fuzzy C-Means clustering algorithm (FCM)}

The FCM proposed by Dunn in 1974 and upgraded by Bezdek et aI., 1987 [24]. has been widely studied and applied [19,25]. FCM is an unsupervised clustering algorithm, as the k-medoids algorithm and $\mathrm{K}$-means. The k-means algorithm is based on hard set but the FCM algorithm is based on non-crisps [25, 26].

The FCM algorithm functions by assigning affiliation to every sensor node corresponding to every cluster center according to the distance between the cluster center and the sensor node. When the sensor nodes are nearestto the cluster center, its memberships in cluster center are the stronger [26, 27]. The FCM algorithm is an iterative optimization algorithm that minimizes the following function.

$$
f=\sum_{i=1}^{n} \sum_{j=1}^{C} u_{i j}^{m}\left\|x_{i}-C H_{j}\right\|^{2}
$$

Where, $n$ is The sum of sensor nodes in WSN, $c$ is the number of clusters are created, $x_{i}$ is the $i^{t} h$ sensor node, $C H_{j}$ is the $j^{t} h$ cluster center, $u_{i j}^{m}$ is the degree of membership of the ith sensor node in the $j^{t} h$ cluster, and $m$ is a positive constant super than $1 .\left\|x_{i}-C H_{j}\right\|$ represents the distance between sensor node $x_{i}$ and the cluster center $\mathrm{CH}_{j}$. The degree of membership $u_{i j}^{m}$ and the cluster center $\mathrm{CH}_{j}$ are difined as follows:

$$
\begin{gathered}
u_{i j}=\frac{1}{\sum_{k=1}^{c}\left(\frac{\left\|x_{i}-c_{j}\right\|}{\left\|x_{i}-c_{k}\right\|}\right)^{\frac{2}{m-1}}} \\
C H_{j}=\frac{\sum_{i=1}^{n} u_{i j}^{m} x_{i}}{\sum_{i=1}^{n} u_{i j}^{m}}
\end{gathered}
$$

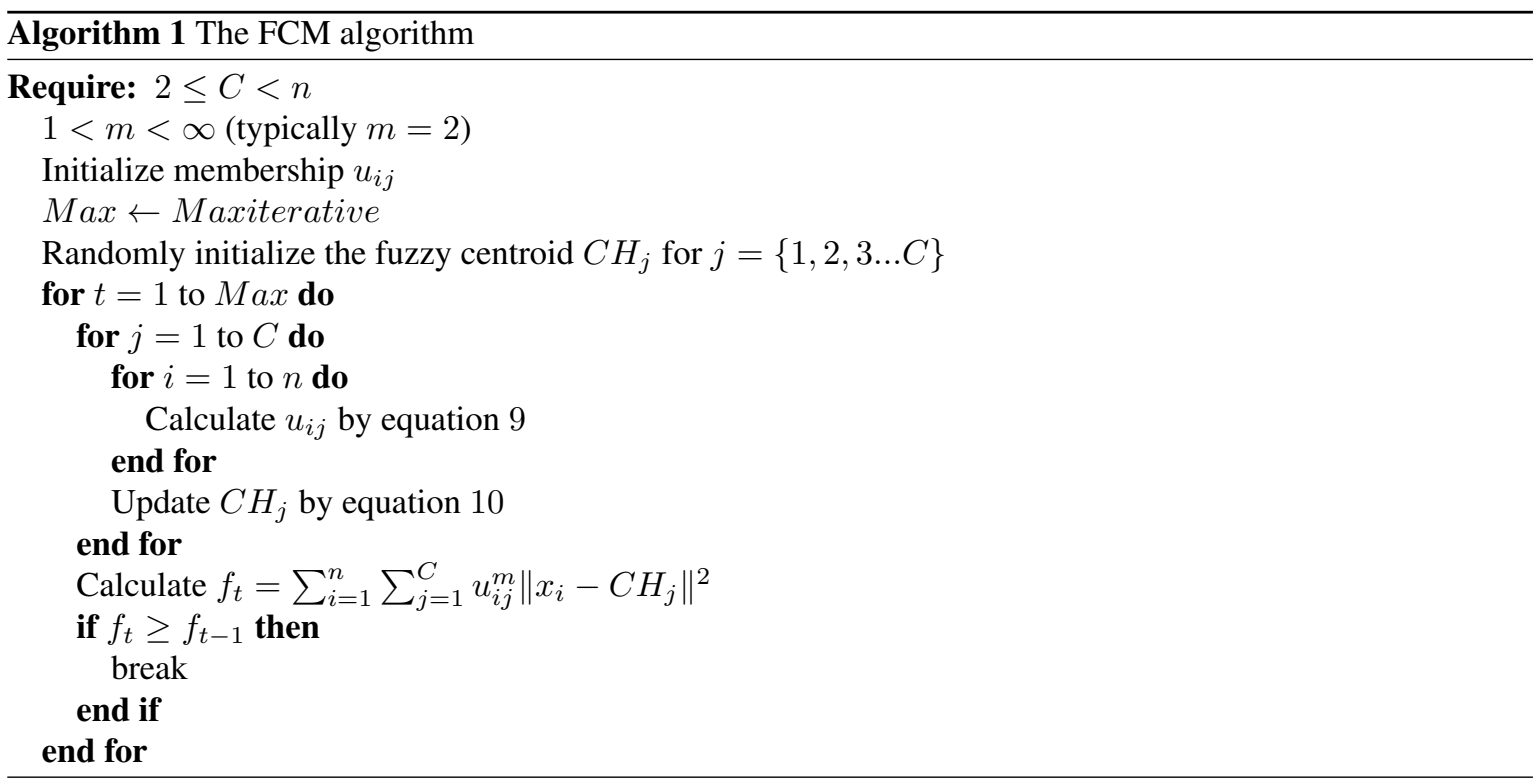

\section{MULTI-ZONAL FUZZY LOGIC HETEROGENEOUS CLUSTERING BASED ON STABLE ELECTION PROTOCOL(FMZ-SEP)}

In this section we discus the majors points about proposed FMZ-SEP. The principal aim of this contribution is to improve network lifetime. The FMZ-SEP protocol is improved version of MZ-SEP.

The static sensor nodes are deployed randomly in an area. The data collected by nodes is forwarded to a BS through the CH. BS is located outside the area. These is not limited in energy and computational power. The $C H$ is selected randomly like the principle of selection of $C H$ in MZ-SEP protocol .

In the FMZ-SEP protocol, the WSN is divided into triangle zones; each zone is considered as small WSN, in order to obtain a better cluster repartition in the WSN.This segmentation improve the lifetime of 
network, the position more than $50 \%$ of $\mathrm{CH}$ as close as possible to the BS. The architecture of network after segmentation is given in the Figure 2. The nodes are not selected as $\mathrm{CH}$; they must be attached to the closest $\mathrm{CH}$ in the network. In FMZ-SEP protocol, the distance between several $\mathrm{CH}$ and the BS is less than or equal to $d_{0}$. This helps to minimize the energy consumption of $\mathrm{CH}$.

The first phase in the FMZ-SEP protocol is to split the area into virtual zones in order to get a better clusters. ALL The zones created in the form of letter V inspired by PSO algorithm. The FMZ-SEP protocol only attempts to find the global minimum between $\mathrm{CH}$ and its members according to the equation 6 . The creation of the zones starts at the nearest point at BS. The Figure 2 shows the division of WSN into multiple zones.

$$
d\left(n_{i}, C H_{r}\right)=\sqrt{\left(n_{i}-C H_{r}\right)^{2}}
$$

Where $n_{i}$ is a node not selected, $\mathrm{CH}_{r}$ is a node become $\mathrm{CH}$.

The zones creation is based on the following keys:

(a) The geographic coordinates of the BS;

(b) $d_{0}$;

(c) The geographic coordinates of deployment area.

$$
d_{0}=\sqrt{\frac{\xi_{f s}}{\xi_{a m p}}}
$$

Where $\xi_{f s}$ is constant corresponding transition from direct path, $\xi_{a m p}$ is constant corresponding transition from multi-path.

Frequently, the FMZ-SEP protocol divides the WSN into 3 zones. All zones have the same vertex point, which is the closest point beteew the WSN and BS.

The first zone is on the right of the BS, where the distance between the BS and each nodes of this zone is less or equal $d_{0}$. The second zone is located to the left of the BS, the nodes of this zone are closer to the $\mathrm{BS}$, for example the distanced node of the BS is located at an equal distance or less than $d_{0}$. The third zone is between the two previous zones.

In our studied example, the dimensions of the area is $100 \mathrm{~m} x 100 \mathrm{~m}$, the geographic coordinates of the BS are $(50,-50)$.

The amount of energy consumed for each node in the right zone or the left zone is calculated by the equation 8 [10]

$$
E_{T x-f s}(k, d)=K\left(E_{\text {elec }}+\xi_{f s} d^{2}\right) \quad \text { if } \quad d<d_{0}
$$

The distances of nodes which belong to zone in the middle of the surveillance field are greater than $d_{0}$ or less or equal to d0. The energy consumed by the $C H$ in the middle zone is calculated by the equations 9 and $10[10]$.

The energy expended in the transmit electronics for free space propagation $E_{T x-f s}$ is described by:

$$
E_{T x-f s}(k, d)=K\left(E_{\text {elec }}+\xi_{f s} d^{2}\right) \quad \text { if } \quad d<d_{0}
$$

The energy expended in the transmit electronics for free multi-path propagation $E_{T x-m p}$ is given by:

$$
E_{T x-m p}(k, d)=K\left(E_{\text {elec }}+\xi_{a m p} d^{4}\right) \quad \text { if } \quad d>=d_{0}
$$

After the zones were formed by the $B S$, we applied the SEP protocol only in zone left and zone right.

In zone middle,before applying the SEP protocol; we determine the optimal cluster number value via the the subtractive clustering algorithm, then we create large clusters through the execution of the FCM algorithm. The clusters are considered as sub-WSN. The SEP protocol is applies in each cluster to construct the small clusters; the first operation is selected the $\mathrm{CHs}$ based on two weighted election probability $P_{\text {normal }}$ and $P_{a d v}$ and their members. The flowchart of the cluster formation process of the FMZ-SEP is showing in the Figure 3. 


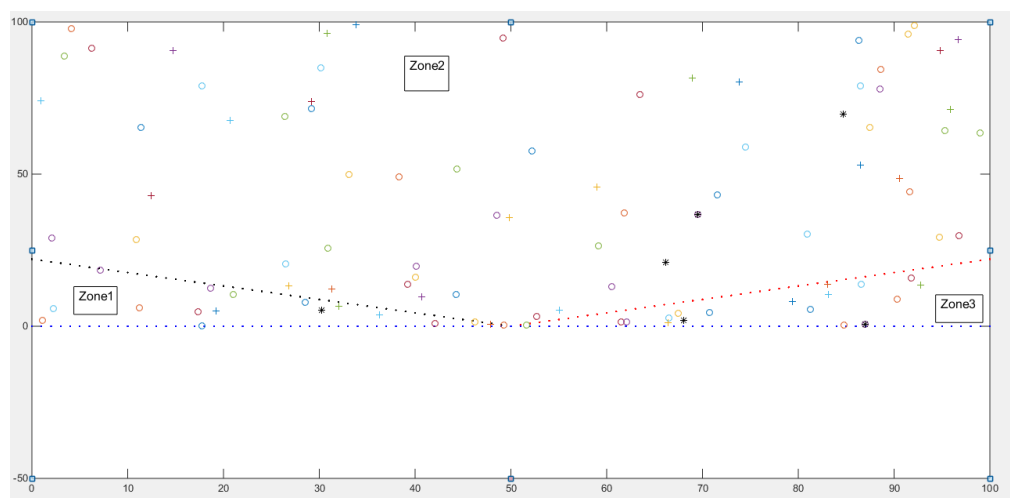

Figure 2. Formation of triangles zones of FMZ-SEP

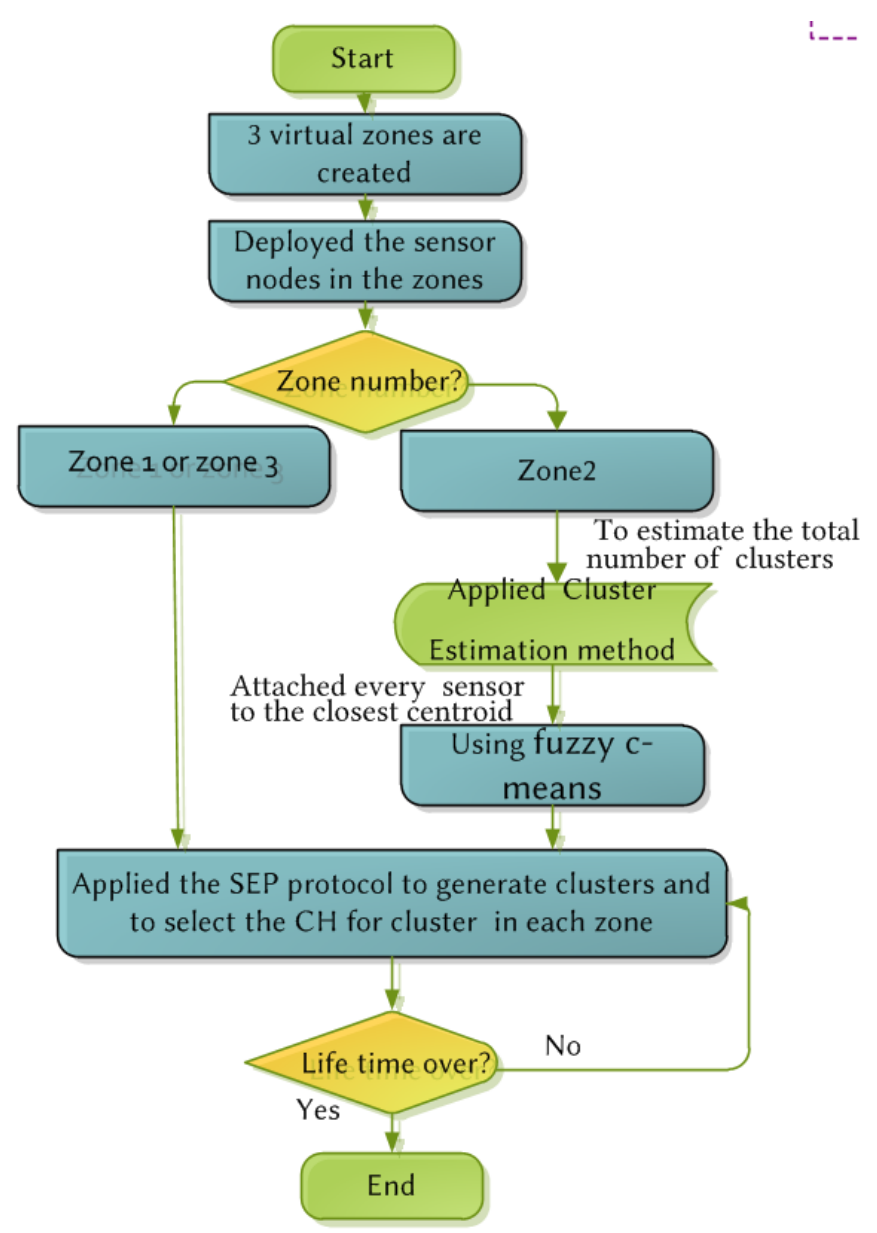

Figure 3. Flowchart of the cluster formation process of FMZ-SEP

\section{SIMULATIONS AND RESULTS}

To study the performance of MZF-SEP protocol, we need to simulate this protocol and compare the results with other protocols of the same category such as the SEP protocol or the MZ-SEP protocol. In this simulation, we adopted the several parameters to mount the scenario of the simulation. The first parameters are: 
(a) The dmentions of our WSN is $100 m x 100 \mathrm{~m}$.

(b) The position of the BS that is inside the WSN or in the outside; in our case the BS is located outside the WSN at the following geographic strings $(50 \mathrm{~m},-50 \mathrm{~m})$.

(c) The number of nodes deployed in the WSN is 100 .

Other parameters are the power consumption model, the size of the message sent to $\mathrm{CH}$ in each round. All simulations are tested in MATLAB.

In simulation, we use the same energy consumption model of SEP protocol. The 100 nodes are randomly deployed in the $100 \mathrm{~m} * 100 \mathrm{~m}$ area. the size of the message transmed by the node is 4000 bit. Figure 2 presents the study WSN. The important parameters of simulation are given in Table 1.

Table 1. Simulation parameters

\begin{tabular}{cc}
\hline Parameter & Value \\
\hline Simulation Area & $100 \mathrm{~m} 100 \mathrm{~m}$ \\
BS Location & $(50 \mathrm{~m},-50 \mathrm{~m})$ \\
Number of Nodes & 100 \\
Transmission Energy $E_{T x}$ & $10 * 10^{-12} \mathrm{~J} / \mathrm{bit} / \mathrm{m}^{2}$ \\
Receiving Energy $E_{R X}$ & $0.0013 * 10^{-12} \mathrm{~J} / \mathrm{bit}_{\mathrm{m}} \mathrm{m}^{4}$ \\
Data Aggregation Energy & $5 * 10^{-9} \mathrm{~J} / \mathrm{bit} / \mathrm{message}$ \\
Transmission Energy $E_{T x}$ & \\
Receiving Energy $E_{R X}$ & $50 * 10^{-9} \mathrm{~J}$ \\
\hline
\end{tabular}

\subsection{Performance parameters}

To evaluate our protocols, we use the following performance parameters:

Stability Period: is the time between the beginning of the operation of WSN and the exhaustion of energy of the first node in WSN. It is the time where all the nodes can send data to $\mathrm{CH}$.

Instability Period: begins just after the end of the period of stability and this duration until the end of operation of the WSN; where all the nodes not have the energy.

Number of alive nodes: the total number of nodes having having sufficient energy.

Simulation results were obtained after running the proposed algorithm several times. The results demonstrate the superiority of the proposed FMZ-SEP algorithm in follwing parametrs :enlargement of stability period, to broaden out the network lifetime and optimization of energy consumption of the entire network.

\subsection{Network lifetime}

Figure 4 shows the results for the total number of dead nodes with respect to operational iterative rounds and without distinction between nodes type. Figure 5 displays the results for the total number of normal dead nodes with respect to operational iterative rounds, and Figure 6 visualizes the results for the total number of advanced dead nodes with respect to operational iterative rounds.

In the three Figures 4, 5 and 6 we observe that our model FMZ-SEP algorithm gives really impressive performance results, both in terms of stability period and instability period. In Figure 4, 5 and 6 the stability period of our model FMZ-SEP algorithm is more than double that of SEP protocol and the MZ-ESP protocol; more by $50 \%$ than the FSEP protocol.

According to Figure 4, the first node is dead at 2458 rounds, 1543 rounds, 924 rounds and 865 rounds for our model FMZ-SEP , FSEP protocol, MZ-ESP protocol and SEP protocol respectively. In Figure 5 the time of stability period is 2457 rounds , 1542 rounds, 924 rounds and 864 rounds for our model FMZ-SEP , FSEP protocol, MZESP protocol and SEP protocol respectively. From Figure 6, the first advanced node dead at 2931 rounds, 2090 rounds, 1456 rounds and 1415 rounds for our model FMZ-SEP, FSEP protocol, MZESP protocol and SEP protocol respectively. The network energy quantity per round is depicted in Figure 7. 


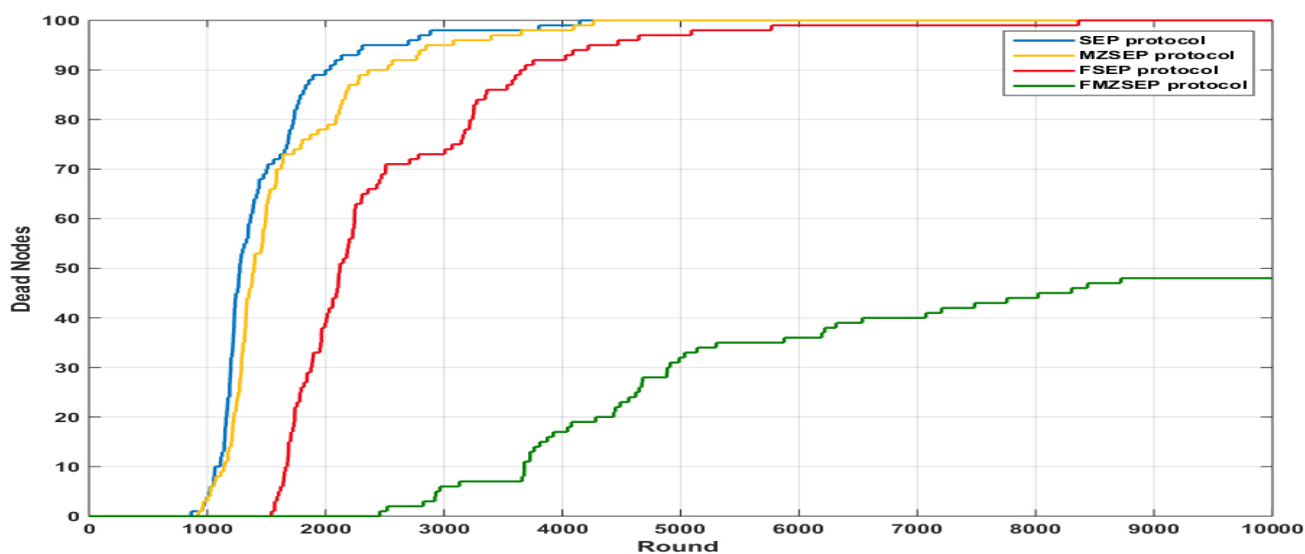

Figure 4. Number of dead nodes per round

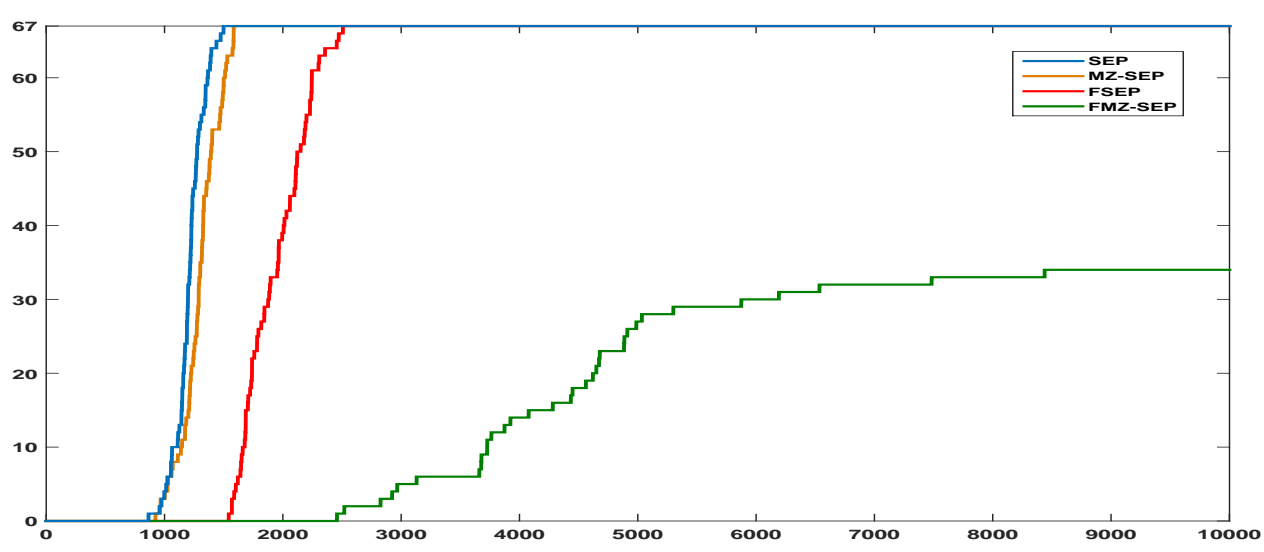

Figure 5. Number of normal dead nodes per round

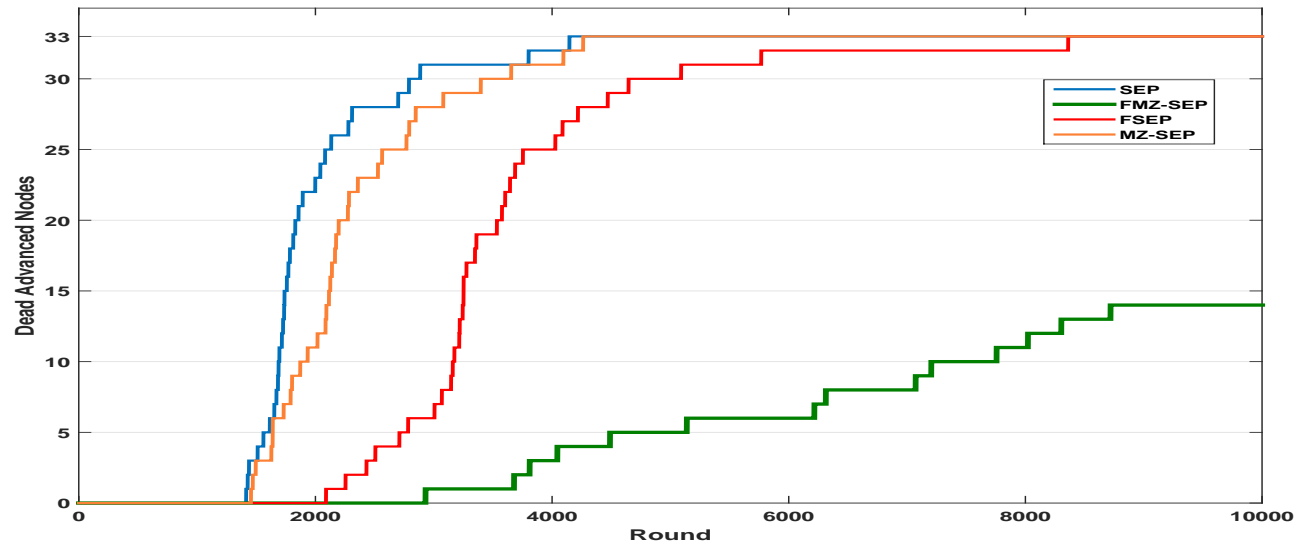

Figure 6. Number of advance dead nodes per round 


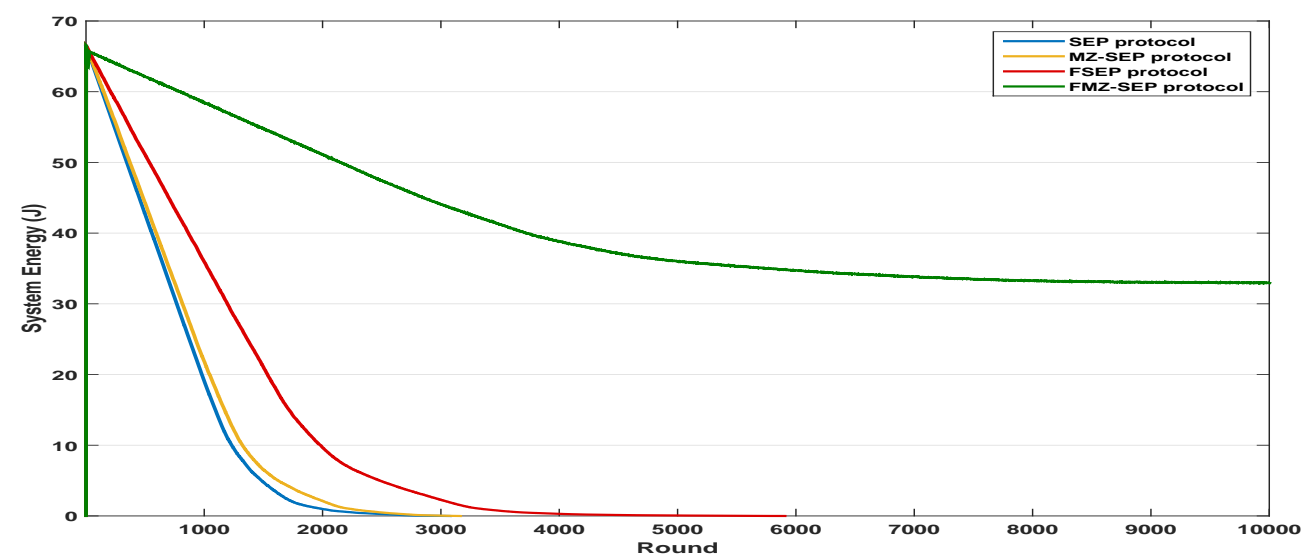

Figure 7. Network energy quantity per round

\subsubsection{MZ-SEP}

The first node is dead at 924 rounds and 865 rounds for MZESP protocol and SEP protocol respectively, the last node is dead at 4147 rounds and 4263 rounds. The stability period of the MZ-SEP protocol is over the $10 \%$ than SEP protocol, the instability period of the MZ-SEP protocol is improved as compared to the SEP.

\subsubsection{FSEP}

According to Table 2, the stability region is 865 rounds, 924 rounds and 1543 rounds and 490 rounds for SEP, MZ-SEP and FSEP respectively. On the other hand, the instability period till, 4147, 4263 and 8362 for SEP, MZ-SEP and FSEP respectively. The results show that the stability region and the instability period are double elongated in case of the FSEP compared to the SEP or MZ-SEP, On the other hand, the overall life time of the FSEP outperforms all the other protocols (SEP and MZ-SEP). The FSEP gives the better results compared to the SEP protocol and MZ-SEP.

Table 2. Percentage of dead nodes per rounds

\begin{tabular}{clllllll}
\hline Dead node & 1 & $10 \%$ & $20 \%$ & $50 \%$ & $70 \%$ & $90 \%$ & $100 \%$ \\
\hline SEP & 865 & 1064 & 1159 & 1275 & 1499 & 1999 & 4147 \\
MZ-SEP & 924 & 1139 & 1223 & 1392 & 1585 & 2359 & 4263 \\
FSEP & 1543 & 1661 & 1739 & 2120 & 2507 & 3645 & 8362 \\
MZF-SEP & 2458 & 3679 & 4282 & 10000 & & & \\
\hline
\end{tabular}

\subsubsection{FMZ-SEP}

Performance results show that model FMZ-SEP was good for improving Stability Period and instability period. The stability period of the FMZ-SEP protocol is over the $150 \%, 150 \%$, and $50 \%$ than SEP protocol ,MZ-ESP protocol and FSEP protocol respectively. In addition, the instability period of the FMZ-SEP protocol is very much improved as compared to the SEP protocol,the MZ-ESP protocol and the FSEP protocol. Then the FMZ-SEP approach reduces the energy consumption by round, and extend the network lifetime.Therefore the FMZ-SEP approach provided the longest lifetime of WSN due to FSEP protocol, MZ-ESP protocol and SEP protocol.

\section{CONCLUSION}

The main objective of this work is to propose a new Hybrid routing protocol based on multiple triangle zones distribution, the subtractive clustering method,fuzzy means and SEP protocol applied for wireless sensor networks. The proposed approach minimizes the energy consumption, extends the network lifetime of the sensor nodes. The evolution and enhancement of the presented routing algorithms should be done in the future. 


\section{REFERENCES}

[1] P. K. Kashyap and S. kuma, "A survey on energy efficient coverage protocols in wireless sensor networks," International Journal of Electrical and Computer Engineering (IJECE), vol. 9, no. 2, pp. 1168-1183, 2019.

[2] I. Ez-zazi, M. Arioua, A. el Oualkadi, and Y. el Assari, "Performance analysis of efficient coding schemes for wireless sensor networks," in RFID And Adaptive Wireless Sensor Networks (RAWSN), 2015 Third International Workshop on. IEEE, 2015, pp. 42-47.

[3] A. J. Swati and R. Priyanka, "Wireless sensor network (wsn): Architectural design issues and challenges," International Journal on Computer Science and Engineering, vol. 2, no. 09, pp. 3089-3094, 2010.

[4] I. Ez-zazi, M. Arioua, A. El Oualkadi, and P. Lorenz, "On the performance of adaptive coding schemes for energy efficient and reliable clustered wireless sensor networks," Ad Hoc Networks, vol. 64, pp. 99-111, 2017.

[5] M. Hammoudeh and M. Arioua, "Sensors and actuators in smart cities," 2018.

[6] A. Mahboub, M. Arioua, E. M. En-Naimi, and I. Ez-zazi, "Performance evalution of energy-efficient clustering algorithms in wireless sensor networks." Journal of Theoretical \& Applied Information Technology, vol. 83, no. 2, 2016.

[7] M. Arioua, Y. El Assari, I. Ez-Zazi, and A. El Oualkadi, "Multi-hop cluster based routing approach for wireless sensor networks," Procedia Computer Science, vol. 83, pp. 584-591, 2016.

[8] A. Mahboub, E. M. En-Naimi, M. Arioua, and H. Barkouk, "Distributed energy efficient clustering algorithm based on fuzzy logic approach applied for heterogeneous wsn," in Proceedings of the 2nd International Conference on Computing and Wireless Communication Systems. ACM, 2017, p. 38.

[9] A. Mahboub, M. Arioua, E. M. En-Naimi, and I. Ezzazi, "Multi-zonal approach for clustered wireless sensor networks," in 2nd International Conference on Electrical and Information Technologies, ICEIT, 2016, pp. 4-7.

[10] W. R. Heinzelman, A. Chandrakasan, and H. Balakrishnan, "Energy-efficient communication protocol for wireless microsensor networks," in System sciences, 2000. Proceedings of the 33rd annual Hawaii international conference on. IEEE, 2000, pp. 10-pp.

[11] A. Mahboub, M. Arioua, I. Ez-Zazi, A. El Oualkadi et al., "Multi-zonal approach clustering based on stable election protocol in heterogeneous wireless sensor networks," in Information Science and Technology (CiSt), 2016 4th IEEE International Colloquium on. IEEE, 2016, pp. 912-917.

[12] A. Mahboub, M. Arioua et al., "Energy-efficient hybrid k-means algorithm for clustered wireless sensor networks," International Journal of Electrical and Computer Engineering (IJECE), vol. 7, no. 4, pp. 2054-2060, 2017.

[13] G. Smaragdakis, I. Matta, and A. Bestavros, "Sep: A stable election protocol for clustered heterogeneous wireless sensor networks," Boston University Computer Science Department, Tech. Rep., 2004.

[14] N. Mittal and U. Singh, "Distance-based residual energy-efficient stable election protocol for wsns," Arabian Journal for Science and Engineering, vol. 40, no. 6, pp. 1637-1646, 2015.

[15] S. Hassan, M. S. Nisar, and H. Jiang, "Dtre-sep: A direct transmission and residual energy based stable election protocol for clustering techniques in hwsn," in Communication Software and Networks (ICCSN), 2015 IEEE International Conference on. IEEE, 2015, pp. 266-271.

[16] N. Mittal, U. Singh, and B. S. Sohi, "A stable energy efficient clustering protocol for wireless sensor networks,” Wireless Networks, vol. 23, no. 6, pp. 1809-1821, 2017.

[17] A. Kashaf, N. Javaid, Z. A. Khan, and I. A. Khan, “Tsep: Threshold-sensitive stable election protocol for wsns," in Frontiers of Information Technology (FIT), 2012 10th International Conference on. IEEE, 2012, pp. 164-168.

[18] R. Logambigai, S. Ganapathy, and A. Kannan, "Energy-efficient grid-based routing algorithm using intelligent fuzzy rules for wireless sensor networks," vol. 68, 2018, pp. 62 - 75. [Online]. Available: http://www.sciencedirect.com/science/article/pii/S0045790617319146

[19] D.-W. Kim, K. Lee, D. Lee, and K. H. Lee, "A kernel-based subtractive clustering method," Pattern Recognition Letters, vol. 26, no. 7, pp. 879-891, 2005.

[20] O. Castillo, P. Melin, J. Kacprzyk, and W. Pedrycz, “Type-2 fuzzy logic: theory and applications," pp. 145-145, 2007.

[21] N. K. Kasabov, Evolving connectionist systems: the knowledge engineering approach. Springer Science \& Business Media, 2007. 
[22] D. Goyal and M. R. Tripathy, "Routing protocols in wireless sensor networks: A survey" in Advanced Computing \& Communication Technologies (ACCT), 2012 Second International Conference on. IEEE, 2012, pp. 474-480.

[23] L.-C. Cheng and H.-A. Wang, "A fuzzy recommender system based on the integration of subjective preferences and objective information," vol. 18, 2014, pp. 290 - 301. [Online]. Available: http://www.sciencedirect.com/science/article/pii/S1568494613002937

[24] Z. Fan, J. Gou, C. Wang, and W. Luo, "Fuzzy model identification based on fuzzy-rule clustering and its application for airfoil noise prediction," Journal of Intelligent \& Fuzzy Systems, vol. 33, no. 3, pp. 1603-1611, 2017.

[25] M. Chen and S. A. Ludwig, "Particle swarm optimization based fuzzy clustering approach to identify optimal number of clusters," Journal of Artificial Intelligence and Soft Computing Research, vol. 4, no. 1, pp. 43-56, 2014.

[26] K. Kapitanova, S. H. Son, and K.-D. Kang, "Using fuzzy logic for robust event detection in wireless sensor networks," Ad Hoc Networks, vol. 10, no. 4, pp. 709-722, 2012.

[27] Q.-T. Lam, M.-F. Horng, J.-N. Lin, J.-P. Hsu et al., "A high energy efficiency approach based on fuzzy clustering topology for long lifetime in wireless sensor networks," in Advanced Methods for Computational Collective Intelligence. Springer, 2013, pp. 367-376.

\section{BIOGRAPHIES OF AUTHORS}

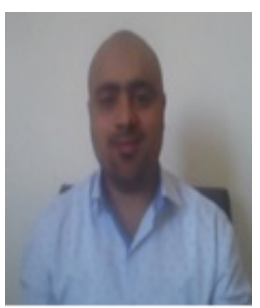

Aziz Mahboub is a $\mathrm{PhD}$ student in LIST (Laboratoire d'Informatique Systèmes et Télécommunications) Department of Computer Science. Faculty of Sciences and Techniques, Tangier, Morocco, at the University of Abdelmalek Essaâdi. He obtained Master Degree in computer science from Faculty of Sciences, University of Abdelmalek Essaadi in 2008. His researches are in fields of wireless sensor networks, Internet of things, wireless communications and mobile computing. He has served as invited reviewer. He has published research papers in IJECES journal ,JATIT journal, conference proceedings as well as chapters of books.

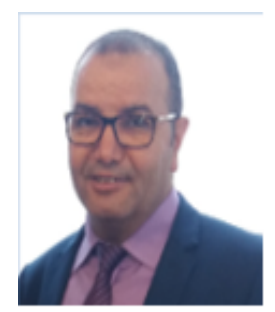

El Mokhtar En-Naimi is a Professor in Faculty of Sciences and Technologies of Tangier, Department of Computer Science. He is responsible for a Bachelor of Sciences and Technologies, BST Computer. Actually, he is a Head of Computer Science Department, since October 2016. He is also a member of the LIST Laboratory (Laboratoire d'Informatique, Systèmes et Télécommunications), the University of Abdelmalek Essaâdi, FST of Tangier, Morocco. He is an Autor and/or Co-Autor of several Articles in Computer Science, in particular, in SMA, Cases Based Reasoning (CBR), eLearning, Big DATA, Datamining, Wireless sensor network, VANet, MANet, etc. In addition, he is an Associate Member of the ISCN - Institute of Complex Systems in Normandy, the University of the Havre, France, since 2009.

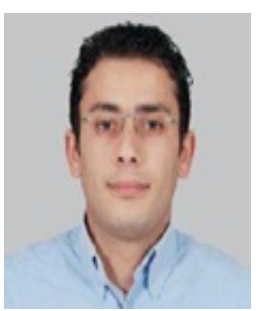

Mounir Arioua received his $\mathrm{PhD}$ degree in Telecommunications and Computer Science from Cadi Ayyad University of Marrakech in 2012. In 2012, he joined the National School of Applied Sciences of Marrakech as research assistant and assistant professor. Currently, he is a Professor at National School of Applied Sciences in the department of Engineering Technologies, University of Abdelmalek Essaadi. His research interests include wireless sensor networks, Internet of things, wireless networking and communication, wireless communications and mobile computing, real-time processing and embedded systems.

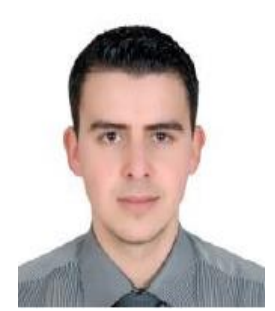

Younes El Assari received M.S. degree from Abdelmalek Essaâdi University in 2013. He is currently a PhD student at the Department of Information and Communication Technologies at National School of Applied Sciences of Tangier, Abdelmalek Essaâdi University, Morocco. His research interests include wireless sensor networks, routing algorithms, Internet of things and software development. 


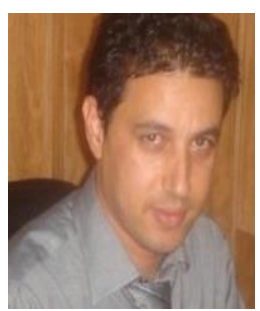

Hamid Barkouk is a $\mathrm{PhD}$ student in LIST (Laboratoire d'Informatique Systèmes et Télécommunications) Department of Computer Science. Faculty of Sciences and Techniques, Tangier, Morocco, at the University of Abdelmalek Essaâdi. His research interests include wireless sensor networks, wireless networking and communication. He is an Author of several articles.

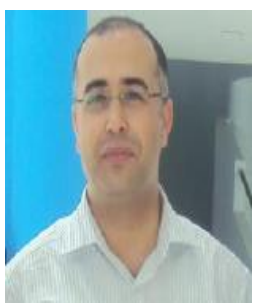

Ahmed El Oualkadi received PhD degree from Poitiers University, France, in 2004. From 2000 to 2003, he was a research assistant at the National Higher Engineering School of Poitiers. In 2004, he was an assistant professor at Institute of Technology, Angoulme. In 2005, he joined the Catholic University of Louvain, Belgium. Currently, he is a professor at national school of applied sciences of Tangier, Abdelmalek Essaadi University. His main research interest is RFIC design for wireless communication, wireless sensor networks, embedded system applications and information technology. 